\title{
Why Is Accessibility So Hard?
}

\section{I sometimes think that, of all the disciplines, ours ought to be the most effective at adapting to the varied needs of users, including those that are challenged to interact with computing}

systems in one way or another. From low to no vision, deafness or hearing loss to carpal tunnel syndrome and various other physical limitations, we really should be able to configure our software to adapt. And in many cases, some very useful, clever, and generalpurpose software adaptations have been achieved. But the problem persists, and it is still not the case that one can hold high expectations of accessible adaptation for a random application that happens to become necessary or, at least, of high interest.

I think I understand some of the problem, but this column is an attempt to begin a dialogue about improving the state of accessibility in our field. This is not only important from the purely ethical perspective, but it is also pragmatic given the demographics of our society and the increasing incidence of need for accessible applications. We are an aging society and we are welcoming home many wounded warriors with the need for assistive response, to mention only two obvious beneficiary groups. One reason this seems to be so hard is that software has unlimited variations and interfaces to applications can take virtually any form. Moreover, we are extending the modalities of interaction to include speech, gestures, mice, touchscreens, other "pointers," keyboards, and so on. We have Web-based applications that take advantage of a wide range of presentation and interaction choices. Not all applications take into account the need to offer distinct and configurable user interfaces and even when some or many such adaptations are offered, some work a lot better than others. The other side of this equation is that the users also manifest unlimited variations in their abilities and it seems unlikely that programmers can be fully cognizant of the nuances of each.

Another theme is the proliferation of platforms through which we may interact with computer-based services and applications. It becomes increasingly difficult to design in detail every mode of interaction, including accessibility variations, for every platform and application imaginable. And even if our imaginations were that good, someone is bound to invent a new application requiring assistive responses that we have not thought about before.

One popular tactic has been to try to create general-purpose tools such as screen readers to assist blind users or automatic captions to help deaf users. Another tactic is to "parameterize" the interfaces so users can pick and choose the variations best suited to their needs. In my experience, the range of parameters devised is fairly large and it is easy to get lost in selecting configurations or even anticipating how well they will fit user needs. Still, these tactics seem important for practitioners to apply when appropriate. The challenges strike me as fundamental, given the range of needs and potential interface designs.

This is by no means a new problem. There cannot be much debate that programmers and user interface (UI) and experience (UX) experts need to think fairly broadly and deeply about potential use cases before settling on an interface design. While the use of libraries intended to confer "accessibility" on arbitrary applications may be helpful, it seems to me that no amount of automatic adapting will make a poorly designed interface accessible. For some of the same reasons that security ought to be "built in" to the initial design, so should accessibility. If UI designers had to try their designs while blindfolded or use their applications with the sound off, they might gain insights into the nuanced demands that accessibility places on good design.

One feature of good interface design is anticipating what the user is likely to need to do next and to prepare for that. A similar notion might inform thinking about accessibility. One is struck by the seemingly impossible challenge faced by blind users and UI designers for them. In the Web-based world, two-dimensional displays, touchscreens, popup windows, drop-down menus, color highlighting, and other signals seem utterly out of reach. One must think how a user interface will behave when it is serialized for audible presentation. In addition, consistency of format and audio feedback from screen to screen also seems like a helpful philosophy.

I would like very much to hear from ACM members, SIGs interested in this space, UX design experts, as well as users of accessibility features about their experiences and their ideas. ${ }^{a}$ Somehow we must find ways to approach this problem with a richer combination of design principles, pragmatic tactics, and artful implementations than we have in hand today. a I also recommend ACM's Transactions on
Accessible Computing as a valuable resource.

Vinton G. Cerf is Vice President and Chief Internet Evangelist at Google Inc. and the president of ACM.

(c) 2012 ACM 0001-0782/12/11 $\$ 15.00$ 